\title{
Improved Understanding of Material Behavior using Correlative In-situ Techniques
}

\author{
Megan J. Cordill ${ }^{1}$, Josef Kreith ${ }^{1}$, Marcel Winhold ${ }^{2}$, Michael Leitner ${ }^{2}$ and Christian H. Schwalb ${ }^{2}$ \\ 1. Erich Schmid Institute of Materials Science, Austrian Academy of Sciences, and Department of \\ Materials Physics, Montanuniversität Leoben, Leoben, Austria. \\ 2. GETec Microscopy GmbH, Vienna, Austria.
}

In order to advance flexible electronic technologies it is important to study the combined electromechanical properties of thin metal films on polymers substrates under mechanical load. Ductile films and lines are an integral part of flexible electronics because they allow current flow between semiconducting islands and other operating features. When ductile films on polymer substrates are strained in tension the substrate can suppress the catastrophic failure that allows for their use in flexible electronics and sensors. However, the charge carrying ductile films must be of an optimum thickness and microstructure for the suppression of cracking to occur [1,2]. In order to improve mechanical and electrical properties of these complex material systems, more work at characterizing the processingstructure-property relationships should be performed. Studies of strained films on polymer substrates tend to emphasize only the electrical properties and thickness effects more than the role of film microstructure or deformation behavior. The microstructure of the film not only determines the mechanical behavior but also influences the electrical behavior and could be optimized if studied in connection with the mechanical behavior.

To address both the electro-mechanical and deformation behavior of metal films supported by polymer substrates, in-situ 4 point probe resistance measurements were performed with in-situ atomic force microscopy (AFM) imaging of the film surface during straining with a novel high-vacuum AFM, the AFSEM (Figure 1). The 4 point probe resistance measurements allow for the examination of the changes in resistance with strain, while the unique design of the AFSEM permits surface imaging and the visualization of localized thinning and crack formation. From the surface images, the crack and deformation densities can be determined using the crack depth to film thickness ratio (Figure 2) [1,2]. AFM is the only technique that can provide the necessary resolution of the deformed film surfaces. Through the correlation of the increase in resistance and the measured deformation density (Figure 3), it was determined that the electrical behavior is only influenced when the deformation density reaches saturation and is an indication of through thickness crack formation. The combination of electrical measurements and surface imaging allow for a complete picture of electro-mechanical behavior needed for the improvement and future success of flexible electronic devices.

\section{References:}

[1] MJ Cordill, VM Marx, MRS Proc. (2013) p. 1.

[2] MJ Cordill et al, Microelectron. Eng. 137 (2014), p. 96. 

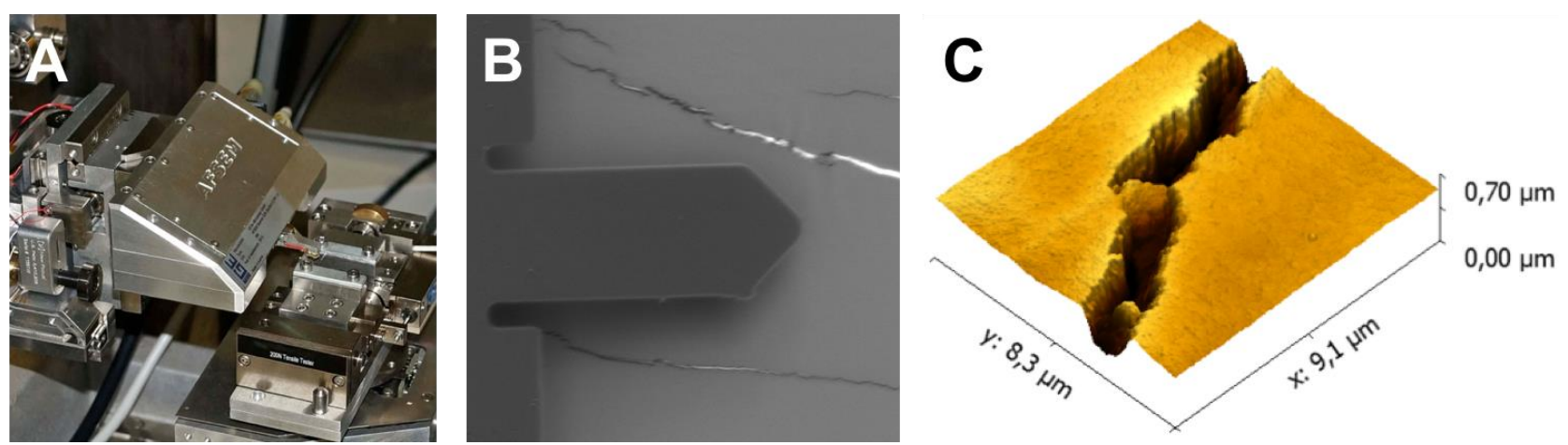

Figure 1. AFSEM's unique design is utilized to perform in-situ straining experiments to directly correlate the changes in electrical resistance and surface damage due to straining. (A) AFSEM and tensie stage installed inside a SEM system. (B) SEM image of cantilever on top of an Inconel/Ag/Teflon film. (C) In situ AFM topography image of crack structure after induced strain.
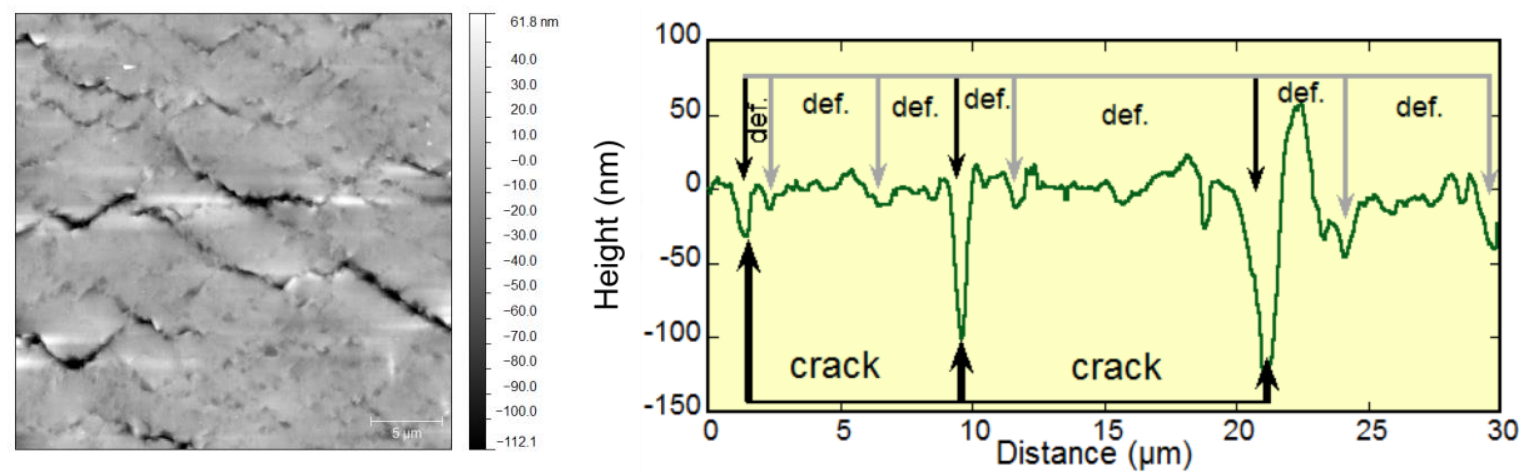

Figure 2. (left) AFM height image of $200 \mathrm{~nm} \mathrm{Cu}$ film on PET strained in-situ under the AFM. (right) Surface profiles extracted from the AFM images with deformation (def.) and cracks indicated [2].

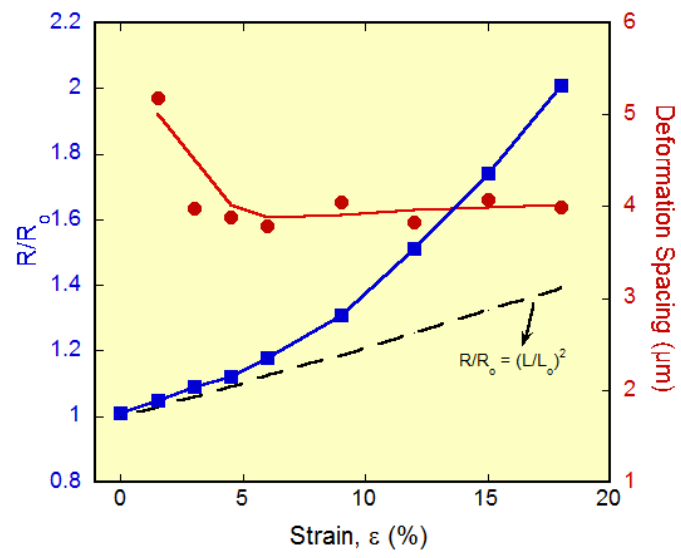

Figure 3. In-situ-squared results allow for the direct comparison of electrical resistance and deformation as a function of strain [2]. 